\title{
Robust Control of Particle Size Distribution in Aerosol Processes
}

\author{
Z. Xiang
}

\author{
Zhengrong Xiang \\ School of Automation \\ Nanjing University of Science and Technology \\ Nanjing, 210094, People's Republic of China \\ E-mail: xiangzr@mail.njust.edu.cn
}

\begin{abstract}
This paper deals with a comprehensive study on robust control of particle size distribution of fractal agglomerate in aerosol processes with simultaneous chemical reaction, nucleation, condensation and coagulation. Firstly, a general aerosol process is described by population balance and mass and energy balances, which describes the evolution of particle size distribution, continuous phase species and temperature of the aerosol system, respectively. A lognormal moment approximations of the population balance model is then presented. Then, the robust state feedback controller is designed for the aerosol process with some unknown uncertainties, the proposed controller is composed of an nominal control term and a robust control term so that it only ensures the stability of the closed-loop system, but also attenuates the effect of the unknown uncertainties on the system. A high-gain observer is adopted to estimate state variables required in the on-line implementation of the state feedback. Finally, the proposed robust controller is applied to an aerosol process for achieving an aerosol size distribution with desired geometric average particle diameter, the simulation results show the robustness properties of the controller with respect to parametric model uncertainty and unmodeled dynamics.
\end{abstract}

Keywords: particle size distribution, aerosol process, population balance, nonlinear systems, robust control

\section{Introduction}

Aerosol process is of interest in many problems of ecology, atmospheric physics, and mechanics of multiphase system[1-3]. The evolution of an aerosol size distribution is modeled by population balance equations, which give rise to nonlinear partial integro-differential equation systems. The nonlinearities usually arise from complex reaction, nucleation, condensation and coagulation rates and their nonlinear dependence on temperature. A variety of solution techniques have been developed to address the complexity at various levels. In most cases, one needs to resort to numerical solutions. One of the standard numerical techniques is to discretize the population balance equation using finite difference/element methods (see[4-8], and the references cited therein), but these methods suffer from extremely large computational requirements which cannot be accommodated by conventional computers. Sectional models offer a computationally less demanding solution by approximating the continuous size distribution by a finite number of sections within which the particle size distribution (PSD) function is assumed to be constant [9]. On the other hands, under appropriate simplifying assumptions, analytical solutions have been developed to solve the population balance equation [10-13].

Recently, the issue of population balance mode-based feedback control of PSD has received considerable attention [14-16]. Previous work in this area include stability analysis and the application of the conventional control schemes to crystallizers and emulsion polymerization processes [17]. Unfortunately, conventional control schemes perform poorly in the face of severe process nonlinearities, and may even 
lead to destabilization of the closed-loop system. These limitations of conventional control schemes have motivated research efforts towards synthesizing nonlinear model-based feedback controllers on spatiallyhomogeneous aerosol processes with application to size distribution control in continuous crystallizers $[18,19]$, and titania aerosol reactor [20,21].

However, most real aerosol systems have uncertainties include unknown or partially known timevarying process parameters, exogenous disturbance, and unmodeled dynamics. It is well known that the presence of uncertain variables and unmodeled dynamics, if not taken into account in the controller design, may be lead to severe deterioration of the nominal closed-loop performance or even to closedloop instability. Therefore, it is very important thing to study robust control of nonlinear aerosol systems with uncertainty.

This paper focuses on robust control of particle process described by uncertain population balances. The objective is to develop a general method for the synthesis of practically implementable robust nonlinear controller that explicitly handle time-varying uncertain variables (such as unknown process parameters and disturbances) and unmodeled dynamics. The robust nonlinear controller enforces stability in the closed-loop system and attenuation of the effect of uncertain variables on the system, and achieve PSD with desired characteristics. To present robust output feedback control techniques that are applicable to a broad range of aerosol systems, we choose to focus on an aerosol process that involves simultaneous chemical reaction, nucleation, condensation and coagulation rather than a specific aerosol.

The remainder of this paper is organized as follows: In Section 2, a general aerosol process is described by population balance and mass and energy balances, which describes the evolution of particle size distribution, continuous phase species and temperature of the aerosol process, respectively. A lognormal moment approximations of the population balance model is presented. In Section 3, the robust state feedback controller is designed for the aerosol system with some unknown uncertainties including unknown or partially known time-varying process parameters, exogenous disturbance, and unmodeled dynamics, the proposed controller is composed of a robust control term and a nominal control term so that it only ensures the stability of the closed-loop system, but also attenuates the effect of the unknown uncertainties on the system. A high-gain observer is adopted to estimate state variables required in the on-line implementation of the state feedback. In Section 4, the proposed robust controller is applied to a general aerosol process for achieving an aerosol size distribution with desired geometric average particle diameter, the simulation results show the robustness properties of the controller with respect to parametric model uncertainty and unmodeled dynamics. Finally, conclusions are given in Section 5.

\section{Mathematical model of aerosol process}

\subsection{Spatially-homogeneous aerosol process}

Consider a general aerosol process which described by the following nonlinear partial integro-differential equation [22]:

$$
\begin{gathered}
\frac{\partial n(v, t)}{\partial t}+\frac{\partial(G(\bar{x}, v) n)}{\partial v}-I\left(v^{*}\right) \delta\left(v-v^{*}\right) \\
=\frac{1}{2} \int_{0}^{v} \beta(\bar{x}, v-\bar{v}, \bar{v}) n(v-\bar{v}, t) n(\bar{v}, t) d \bar{v}-n(v, t) \int_{0}^{\infty} \beta(v, \bar{v}) n(\bar{v}, t) d \bar{v}
\end{gathered}
$$

where the term $n(v, t)$ represents the PSD function at time $t, v$ is the particle volume, $I\left(v^{*}\right)$ is the nucleation rate, $G(\bar{x}, v)$ and $\beta(\bar{x}, v-\bar{v}, \bar{v})$ are the diffusional condensation growth function and the Brownian coagulation kernel of agglomerates, $\delta(\cdot)$ is the standard Dirac function. 
A mathematical model which predicts the time evolution of the concentrations of species and temperature of the gas phase has the following form [21]:

$$
\frac{d \bar{x}}{\partial t}=f(\bar{x})+g(\bar{x}) u(t)+\bar{A} \int_{0}^{\infty} a(\eta, v, \bar{x}) d v
$$

where $\bar{x}(t)$ is an $\mathrm{n}$-dimensional vector of state variables that depend on time, $\bar{A}$ is constant matrix, $f(\bar{x}), g(\bar{x}), a(\eta, v, \bar{x})$ are nonlinear vector functions and $u(t)$ is the time-varying manipulated input (e.g. wall temperature). The term $\bar{A} \int_{0}^{\infty} a(\eta, v, \bar{x}) d v$ accounts for mass and heat transfer from the continuous phase to all the particles in the population.

The diffusional condensation growth function $G(\bar{x}, v)$ and the Brownian coagulation kernel of agglomerates $\beta(\bar{x}, v-\bar{v}, \bar{v})$, for the free molecule size regimes, are represented by

$$
\begin{gathered}
G_{F M}(\bar{x}, v)=B_{1} v^{1 / 3}(S-1), B_{1}=(36 \pi)^{1 / 3} v_{0} n_{s}\left(\frac{k_{B} T}{2 \pi m_{0}}\right)^{1 / 2}, \\
\beta_{F M}(\bar{x}, v, \bar{v})=B_{2}\left(v^{1 / D_{f}}+\bar{v}^{1 / D_{f}}\right)^{2} \sqrt{\frac{1}{v}+\frac{1}{\bar{v}}}, B_{2}=(3 / 4 \pi)^{2 / D_{f}-1 / 2}\left(6 k_{B} T / \rho\right)^{1 / 2} r^{2-6 / D_{f}}
\end{gathered}
$$

and for the near-continuum and the continuum regimes

$$
\begin{gathered}
G_{c}(\bar{x}, v)=B_{3} v^{1 / 3}(S-1), B_{1}=\left(48 \pi^{2}\right)^{1 / 3} C_{f} v_{0} n_{s}, C_{f}=\frac{\lambda}{3}\left(\frac{8 k_{B} T}{\pi m_{0}}\right)^{1 / 2} \\
\beta_{c}(\bar{x}, v, \bar{v})=B_{2}\left(v^{1 / D_{f}}+\bar{v}^{1 / D_{f}}\right)\left[\frac{C(v)}{v^{1 / D_{f}}}+\frac{C(\bar{v})}{\bar{v}^{1 / D_{f}}}\right], B_{4}=\frac{2 k_{B} T}{3 \mu}
\end{gathered}
$$

In the above equations, $S$ is the saturation ratio, $T$ is the absolute temperature, $D_{f}$ is the fractal (Hausdorff) dimension, $C_{f}$ is the condensable vapor diffusivity, $\lambda$ is the mean free path of the gas ( $\lambda=$ $v \pi M_{w} / 2 k_{\mathrm{B}} T N_{a v}$, where $v$ and $M_{w}$ are the kinematic viscosity and molecular weight of the fluid, respectively, and $N_{a v}$ is the Avogadro's number), $\mu$ is the viscosity of the fluid, $n_{s}$ is the monomer concentration at saturation ( $n_{s}=P_{S} / k_{B} T$, where $P_{S}$ is the saturation pressure), $m_{0}$ is the monomer mass, $v_{0}$ is the monomer volume, $\rho$ is the particle density, $r$ is the particle radius, $C(v)=1+B_{5} \lambda / r$ is the Cunningham slip correction factor, and $B_{5}=1.257$.

The nucleation rate $I\left(v^{*}\right)$ is assumed to follow the classical Becker-Doring theory and is given by the following expression [23]:

$$
I=n^{2} s_{0}\left(\frac{k_{B} T}{2 \pi m_{0}}\right)^{1 / 2} S^{2}\left(\frac{2}{9 \pi}\right)^{1 / 3} \Sigma^{1 / 2} \exp \left(-k^{*} \ln \frac{S}{2}\right)
$$

where $s_{0}$ is the monomer surface area and $k^{*}$ is the number of monomers in the critical size nucleus which is given by:

$$
k^{*}=\frac{\pi}{6}\left(\frac{4^{\Sigma}}{\ln S}\right)^{3}
$$

where $\Sigma=\gamma v_{0}^{2 / 3} / k_{B} T$ and $\gamma$ is the surface tension.

Remark 1. It is found experimentally that in many cases of practical interest, the total number of primary particles $N_{p}$ in an agglomerate is related to characteristic radius $R$ through a power law expression $N_{p} \sim$ $R^{D_{f}}$, where the exponent $D_{f}$ is called the fractal dimension. This is usually true in a statistical sense after averaging over many agglomerates with the same $N_{p}$. The value of $D_{f}$ depends on the details of the agglomerate formation process. For compact agglomerates we have $D_{f} \rightarrow 3$, while for chain-like agglomerates we have $D_{f} \rightarrow 1$. 
Remark 2. It is noticed that the collision kernel $\beta_{F M}$ in (3) is in a form that appears to be rather difficult to expand into a series with a manageable number of terms. The following approximation can be made [11];

$$
\left(\frac{1}{v}+\frac{1}{\bar{v}}\right)^{1 / 2}=b\left(\frac{1}{v^{1 / 2}}+\frac{1}{\bar{v}^{1 / 2}}\right)
$$

The variables for $b$ depend on the initial geometric standard deviation $\sigma_{0}$ and on the fractal dimension $D_{f}$.

Remark 3. The manipulated input $u(t)$ enters system (1)-(2) through (2), this assumption is usually satisfied in most practical applications where the wall temperature is chosen as the manipulated input.

\subsection{Moment model}

In this subsection, we present moment model of size distribution of fractal agglomerates in aerosol processes with simultaneous chemical reaction, nucleation, condensation and coagulation. Many experimental results and numerical calculation indicate that aerosol PSDs can be adequately described by unimodal lognormal functions. This result makes it possible to develop a moment model for the aerosol process in terms of the three leading moments of the size distribution. The log-normal size distribution function is written as:

$$
n(v, t)=\frac{1}{3^{v}} \frac{N(t)}{\sqrt{2 \pi} \ln \sigma(t)} \exp \left[\frac{-\ln ^{2}\left\{v / v_{g}(t)\right\}}{18 \ln ^{2} \sigma(t)}\right]
$$

where $N(t)$ is the total number concentration of particles, $\sigma(t)$ is the geometric standard deviation, and $v_{g}(t)$ is the geometric number mean particle volume. The $k$ th moment of the particle size distribution is written as:

$$
M_{k}(t)=\int_{0}^{\infty} v^{k} n(v, t) d v
$$

where $k$ is an arbitrary real number. According to the properties of a log-normal function, any moment can be written in terms of $M_{1}, v_{g}$ and $\sigma$ as follows

$$
M_{k}=M_{1} v_{g}^{k-1} \exp \left\{\frac{9}{2}\left(k^{2}-1\right) \ln ^{2} \sigma\right\}
$$

Obviously, the three leading moments are sufficient to generate the lognormal PSD. Using the same derivation procedure as the one in [21], we can easily obtain the following equations, which describe the time evolution of the three leading moments (i.e. the zeroth, first and second moments) of the size distribution for the free molecule size.

$$
\begin{gathered}
\frac{d M_{0}}{d t}=I-b B_{2}\left(M_{2 / D_{f}-1 / 2} M_{0}+2 M_{1 / D_{f}} M_{1 / D_{f}-1 / 2}+M_{2 / D_{f}} M_{-1 / 2}\right) \\
\frac{d M_{1}}{d t}=I v^{*}+B_{1}(s-1) M_{2 / D_{f}-1 / 2} \\
\frac{d M_{2}}{d t}=I v^{* 2}+2 B_{1}(s-1) M_{1+2 / D_{f}} \\
+2 b B_{2}\left(M_{2 / D_{f}+1 / 2} M_{0}+2 M_{1+1 / D_{f}} M_{1 / D_{f}+1 / 2}+M_{1+2 / D_{f}} M_{1 / 2}\right)
\end{gathered}
$$

Similar to the case of the free molecule regime, the dynamics of the zeroth and second moments of the aerosol size distribution in the continuum size regime is described by the following equations:

$$
\begin{gathered}
\frac{d M_{0}}{d t}=I-B_{4}\left[M_{0}^{2}+2 M_{1 / D_{f}} M_{-1 / D_{f}}+B_{5} \lambda\left(\frac{4}{3} \pi\right)^{1 / 5}\left(M_{0} M_{-1 / D_{f}}+M_{1 / D_{f}} M_{-1+1 / D_{f}}\right)\right] \\
\frac{d M_{1}}{d t}=I v^{*}+B_{3}(s-1) M_{1 / D_{f}}
\end{gathered}
$$




$$
\begin{gathered}
\frac{d M_{2}}{d t}=I v^{* 2}+2 B_{3}(s-1) M_{1+1 / D_{f}} \\
+2 B_{4}\left[M_{1}^{2}+M_{1+1 / D_{f}} M_{1-1 / D_{f}}++B_{5} \lambda\left(\frac{4}{3} \pi\right)^{1 / 3}\left(M_{1} M_{1-1 / D_{f}}+M_{1 / D_{f}} M_{1+1 / D_{f}}\right)\right]
\end{gathered}
$$

Table 1 Dimensionless variables

$$
\begin{aligned}
& N=M_{0} / n_{s}, \text { Aerosol concentration } \\
& V=M_{1} / n_{s} v_{0} d, \text { Aerosol volume } \\
& V_{2}=M_{2} / n_{s} v_{0}^{2}, \text { Second aerosol moment } \\
& \tau=\left(2 \pi m_{0} / k_{B} T\right)^{1 / 2} / n s_{0} \text {, Characteristic time for particle growth } \\
& K=\left(2 k_{B} T / 3 \mu\right) n_{s} \tau, \text { Coagulation coefficient } \\
& K_{n_{0}}=\lambda / r_{0}, \text { Monomer Knudsen number } \\
& I^{\prime}=I /\left(n_{s} / \tau\right), \text { Nucleation rate } \\
& R_{r}^{\prime}=R_{r} /\left(n_{s} / \tau\right), \text { Reaction rate group } \\
& v_{g}^{\prime}=v_{g} / v_{\mathrm{o}} \\
& r_{g}^{\prime}=r_{g} / r_{\mathrm{O}} \\
& \theta=t / \tau
\end{aligned}
$$

The moment equations for the free molecule size and continuum size regimes may be combined to describe the aerosol dynamics over the entire particle size spectrum by using the harmonic average of the dimensionless coagulation rates in the free molecule size and continuum size regimes [23]. Using the dimensionless variables listed in Table 1, we have to the following equations:

Zeroth moment

$$
\frac{d N}{d \theta}=I^{\prime}-\xi N^{2}
$$

where

$$
\begin{gathered}
\xi=\frac{\xi_{F M} \xi_{c}}{\xi_{F M}+\xi_{c}}, \quad \xi_{F M}=r_{g}^{\prime 1 / 2} b\left[\exp \left(\frac{25}{8} \ln ^{2} \sigma\right)+2 \exp \left(\frac{5}{8} \ln ^{2} \sigma\right)+\exp \left(\frac{1}{8} \ln ^{2} \sigma\right)\right] \\
\xi_{c}=K\left[1+\exp \left(\ln ^{2} \sigma\right)+B_{5}\left(\frac{K_{n_{0}}}{r_{g}^{\prime}}\right) \exp \left(\frac{1}{2} \ln ^{2} \sigma\right)\left(1+\exp \left(2 \ln ^{2} \sigma\right)\right]\right.
\end{gathered}
$$

First moment

$$
\frac{d V}{d \theta}=I^{\prime} k^{*}+\eta(S-1) N
$$

where

$$
\begin{gathered}
\eta=\frac{\eta_{F M} \eta_{c}}{\eta_{F M}+\eta_{c}}, \quad \eta_{F M}=v_{g}^{\prime 2 / 3} \exp \left(2 \ln ^{2} \sigma\right) \\
\eta_{c}=\frac{4 K_{n_{0}}}{3} v_{g}^{\prime 1 / 3} \exp \left(\frac{1}{2} \ln ^{2} \sigma\right)
\end{gathered}
$$

Second moment

$$
\frac{d V_{2}}{d \theta}=I^{\prime} k^{* 2}+2 \varepsilon(S-1) V+2 \zeta V^{2}
$$

where

$$
\begin{gathered}
\varepsilon=\frac{\varepsilon_{F M} \varepsilon_{c}}{\varepsilon_{F M}+\varepsilon_{c}}, \quad \zeta=\frac{\zeta_{F M} \zeta_{c}}{\zeta_{F M}+\zeta_{c}} \\
\varepsilon_{F M}=v_{g}^{\prime 1 / 2} b \exp \left(8 \ln ^{2} \sigma\right), \quad \varepsilon_{c}=\frac{4 K_{n_{0}}}{3} v_{g}^{\prime 1 / 3} \exp \left(\frac{7}{2} \ln ^{2} \sigma\right) \\
\zeta_{F M}=r_{g}^{\prime 1 / 2} b \exp \left(\frac{3}{2} \ln ^{2} \sigma\right)\left[\exp \left(\frac{25}{8} \ln ^{2} \sigma\right)+2 \exp \left(\frac{5}{8} \ln ^{2} \sigma\right)+\exp \left(\frac{1}{8} \ln ^{2} \sigma\right)\right] \\
\zeta_{c}=K\left[1+\exp \left(\ln ^{2} \sigma\right)+B_{5}\left(\frac{K_{n_{0}}}{r_{g}^{\prime}}\right) \exp \left(-\frac{1}{2} \ln ^{2} \sigma\right)\left(1+\exp \left(-2 \ln ^{2} \sigma\right)\right]\right.
\end{gathered}
$$


The rate of change of $S$ can be obtained from a monomer balance and is given by:

$$
\frac{d S}{d \theta}=R_{r}^{\prime} N_{a v}-I^{\prime} k^{*}-\eta(S-1) N
$$

\subsection{Mathematical model of aerosol process}

We consider a typical aerosol process in a cylindrical volume with diameter $D_{T}$. Under the assumption of lognormal aerosol size distribution, the dimensionless model that describes the evolution of the first three moments of the distribution, along with the saturation ratio, reactant concentrations and fluid temperature, takes the following form:

$$
\begin{gathered}
\frac{d N}{d \theta}=I^{\prime}-\xi N^{2} \\
\frac{d V}{d \theta}=I^{\prime} k^{*}+\eta(S-1) N \\
\frac{d V_{2}}{d \theta}=I^{\prime} k^{* 2}+2 \varepsilon(S-1) V+2 \zeta V^{2} \\
\frac{d S}{d \theta}=C \bar{C}_{1} \bar{C}_{2}-I^{\prime} k^{*}-\eta(S-1) N \\
\frac{d \bar{C}_{1}}{d \theta}=-A_{1} \bar{C}_{1} \bar{C}_{2} \\
\frac{d \bar{C}_{2}}{d \theta}=-A_{2} \bar{C}_{1} \bar{C}_{2} \\
\frac{d \bar{T}}{d \theta}= \\
B \bar{C}_{1} \bar{C}_{2} \bar{T}+E \bar{T}\left(\bar{T}_{w}-\bar{T}\right)
\end{gathered}
$$

where $\bar{C}_{1}$ and $\bar{C}_{2}$ are the dimensionless reactant concentrations, $\bar{T}, \bar{T}_{w}$ are the dimensionless fluid temperature and the dimensionless temperature of the heat transferring medium at the system boundary, respectively. $A_{1}, A_{2}, B, C, E$ are dimensionless quantities, their explicit form are given in Table 2 .

Table 2 Dimensionless variables for the model

$$
\begin{aligned}
& A_{1}=\tau k P_{0} y_{20} / R T_{0} \\
& A_{2}=\tau k P_{0} y_{10} / R T_{0} \\
& B=\tau k P_{0} \Delta H_{\tau} y_{10} y_{20} / R T_{0}^{2} C_{p} \\
& C=N_{a v} \tau k y_{10} y_{20}\left(P_{0} / R T_{0}\right)^{2} / n_{s} \\
& E=4 U R T_{0} \tau / D_{T} C_{p} P_{0} \\
& \bar{C}_{i}=y_{i} / y_{i 0} \bar{T} \\
& \bar{T}=T / T_{0} \\
& \bar{T}_{w}=T_{w} / T_{0}
\end{aligned}
$$

We formulate the control problem as one of tracking the geometric average particle diameter of the aerosol system, by manipulating the wall temperature, i.e.

$$
y(t)=d_{p g}(t), \quad u(t)=\bar{T}_{w}(t)-\bar{T}_{w s}
$$

where $\bar{T}_{w s}=T_{w s} / T_{0}=1$.

We write the model (18) in the following form:

$$
\frac{d \tilde{x}}{d t}=\tilde{f}(\tilde{x})+\tilde{g}(\tilde{x}) u(t), y=\tilde{h}(\tilde{x})
$$

where the explicit form of $\tilde{x}, \tilde{f}(x), \tilde{g}(\tilde{x})$ can be obtained by comparing (18) and (19). 
Remark 4. In addition to being highly nonlinear, the real aerosol processes have uncertainties including unknown or partially known time-varying process parameters, exogenous disturbance, and unmodeled dynamics. Therefore, the real aerosol systems can be described by the following systems.

$$
\frac{d \tilde{x}}{d t}=\tilde{f}(\tilde{x})+\Delta \tilde{f}(\tilde{x})+(\tilde{g}(\tilde{x})+\Delta \tilde{g}(\tilde{x})) u(t), y=\tilde{h}(\tilde{x})
$$

where $\Delta \tilde{f}(\tilde{x})$ and $\Delta \tilde{g}(\tilde{x})$ represent uncertainties caused by unknown or partially known time-varying process parameters, exogenous disturbance, and unmodeled dynamics.

\section{Robust controller design}

In this section, we will begin with the design of the robust controller. Our objective is to design a robust output feedback controller which guarantees boundedness of all variables for the closed-loop system and tracking of a given reference signal $y_{d}$.

By differentiating the output $y(t)$ with respect to $t$, we obtain the following nonlinear system represented by the differential equation

$$
\begin{aligned}
& y^{(r)}=f\left(y, y^{(1)}, \cdots, y^{(r-1)}\right)+\Delta f\left(y, y^{(1)}, \cdots, y^{(r-1)}\right)+ \\
& \left(g\left(y, y^{(1)}, \cdots, y^{(r-1)}\right)+\Delta g\left(y, y^{(1)}, \cdots, y^{(r-1)}\right)\right) u
\end{aligned}
$$

where $r$ is the relative order (The definition can be found in [24], and omitted here for brevity) of the output $y(t)$ with respect to the manipulated input $u(t)$.

By taking $x_{1}=y, x_{2}=y^{(1)}$ up to $x_{r}=y^{(r-1)}$, we can represent the augmented system by the state space model

$$
\begin{aligned}
& \dot{x}=A x+B\left(v+D_{1}(x)\right) \\
& y=x_{1}
\end{aligned}
$$

where $x=\left(x_{1}, x_{2}, \cdots, x_{r}\right)^{T}, \quad v=f(x)+g(x) u, \quad \delta_{1}(x)=\Delta g(x) / g(x)$,

$D_{1}(x)=\Delta f(x)+\delta_{1}(x)(v-f(x)),(A, B)$ is controllable canonical pairs of the form

$$
A=\left(\begin{array}{ccccc}
0 & 1 & 0 & \cdots & 0 \\
0 & 0 & 1 & \cdots & 0 \\
\cdots & \cdots & \cdots & \ddots & \vdots \\
0 & 0 & 0 & \cdots & 1 \\
0 & 0 & 0 & \cdots & 0
\end{array}\right) \in R^{r \times r}, B=\left(\begin{array}{c}
0 \\
0 \\
\vdots \\
0 \\
1
\end{array}\right) \in R^{r \times 1}
$$

Assuming the states is available for feedback, taking

$$
\begin{aligned}
& e_{1}=y-y_{r}=x_{1}-y_{r} \\
& e_{2}=\dot{y}-\dot{y}_{r}=x_{2}-\dot{y}_{r} \\
& \vdots \\
& e_{r}=y^{(r-1)}-y_{r}^{(r-1)}=x_{r}-y_{r}^{(r-1)}
\end{aligned}
$$

and $e=\left(e_{1}, e_{2}, \cdots, e_{r}\right)^{T}$, we rewrite (22) as

$$
\dot{e}=A e+B\left(v+D_{1}\left(e+Y_{d}\right)-y_{d}^{(r)}\right)
$$

where $Y_{d}=\left(y_{d}, \dot{y_{d}}, \cdots, y_{d}^{(r-1)}\right)^{T}$. 
Obviously, if $D_{2}(\cdot)=$ o (i.e. $\Delta f(x)=\Delta g(x)=0$ ), we can use the following nominal state feedback control to achieve our objective.

$$
u_{1}=\frac{1}{g(x)}\left(-K e+y_{d}^{(r)}-f(x)\right)
$$

where matrix $K$ is chosen such that the matrix $A_{m}=A-B K$ is Hurwitz, i.e., all its eigenvalues are in the left open plane.

However, due to the presence of uncertainties $\Delta f(x)$ and $\Delta g(x)$, a nominal state feedback control alone cannot ensure the stability of the closed-loop system, it is necessary to add a robust compensator to deal with it.

$$
u_{2}=-\frac{1}{g(x)\left(1-\Delta_{1}(x)\right)} \frac{B^{T} P e \mu^{2}(x)}{\left\|B^{T} P e\right\| \mu(x)+\varepsilon_{1} \exp \left(-\varepsilon_{2} t\right)}
$$

where $\varepsilon_{1}, \varepsilon_{2}>0, P=P^{T}>0$ is a solution of the following Lyapunov equation

$$
\begin{gathered}
P(A-B K)+(A-B K)^{T} P=-Q, \quad \forall Q^{T}=Q>0 \\
\mu(x)=\Delta_{1}(x)\left(\left|y_{d}^{(r)}-K e\right|+|f(x)|\right)+|\Delta f(x)|, \quad \Delta_{1}(x)=\sup _{x \in R^{r}}\left|\delta_{1}(x)\right|
\end{gathered}
$$

The robust state feedback controller we proposed is as follows

$$
u=u_{1}+u_{2}
$$

Theorem 5. Suppose that $\Delta_{1}(x)=\sup _{x \in R^{r}}\left|\delta_{1}(x)\right|<1$, consider system (22) under the robust state feedback controller (26), the closed-loop system have the following properties:

(1) The state of the closed-loop system is bounded.

(2) The output of the closed-loop system satisfy $\lim _{t \rightarrow \infty}\left|y-y_{d}\right|=0$.

Proof: Let

$$
v_{2}=-\frac{1}{\left(1-\Delta_{1}(x)\right)} \frac{B^{T} P e \mu(x)^{2}}{\left\|B^{T} P e\right\| \mu(x)+\varepsilon_{1} \exp \left(-\varepsilon_{2} t\right)}
$$

The closed-loop system under the robust state feedback controller (26) is as follows

$$
\dot{e}=(A-B K) e+B\left(v_{2}+D_{1}(x)\right)
$$

Consider the Lyapunov function candidate

$$
V(e)=e^{T}(t) P e(t)
$$

The derivative of $V(e)$ along the trajectories of the system is given by

$$
\dot{V}(e)=2 e^{T} P(A-B K) e+2 e^{T} P B\left(v_{2}+D_{1}(x)\right)
$$

Obviously, we have

$$
\begin{gathered}
\left|D_{1}(x)\right| \leq|\Delta f(x)|+\Delta_{1}(x)(|v|+|f(x)|) \\
\leq \Delta_{1}(x)\left|v_{2}\right|+|\mu(x)|
\end{gathered}
$$

Therefore

$$
\begin{gathered}
2 e^{T} P B\left(v_{2}+D_{1}(x)\right) \\
\leq-2\left(1-\Delta_{1}(x)\right)\left\|B^{T} P e\right\| \cdot\left|v_{2}\right|+2\left\|B^{T} P e\right\| \mu(x)
\end{gathered}
$$




$$
\leq \frac{{ }_{2} B^{T} P e \mu(x) \varepsilon_{1} \exp \left(-\varepsilon_{2} t\right)}{\left\|B^{T} P e\right\| \mu(x)+\varepsilon_{1} \exp \left(-\varepsilon_{2} t\right)} \leq 2 \varepsilon_{1} \exp \left(-\varepsilon_{2} t\right)
$$

Substituting (29) into (28), we have

$$
\dot{V}(e) \leq-\lambda_{\min }(Q)\|e\|^{2}+2 \varepsilon_{1} \exp \left(-\varepsilon_{2} t\right)
$$

where $\lambda_{\min }(Q)$ denotes the minimum eigenvalue of $Q$. Using the lemma in [25], we have

$$
\lim _{t \rightarrow \infty} e=0
$$

This completes the proof.

The on-line implementation of the controller (26) requires that the values of the state variables $x$ are known. Unfortunately, $x$ may be not be known in many practical applications. One way to address this problem is to use state observer to estimate $x$. Here we use the high-gain state observer as

$$
\begin{aligned}
& \dot{\hat{e}}_{i}=\hat{e}_{i+1}+\frac{\alpha_{i}}{\varepsilon}\left(e_{1}-\hat{e}_{1}\right), \quad 1 \leq i \leq r-1 \\
& \hat{e}_{n}=\frac{\alpha_{n}}{\varepsilon}\left(e_{1}-\hat{e}_{1}\right)
\end{aligned}
$$

where $\hat{e}=\hat{x}-Y_{d}, \hat{x}$ denote the estimate state of the state $x, \varepsilon$ is a small positive parameter, the constants $\alpha_{i}>0$ are chosen such that the roots of

$$
s^{r}+\alpha_{1} s^{r-1}+\cdots+\alpha_{r-1} s+\alpha_{r}=0
$$

have negative real parts.

The state feedback controller (26) and the state observer (31) can be combined to yield the following nonlinear robust output feedback controller:

$$
\begin{gathered}
\dot{\hat{e}}_{i}=\widehat{e}_{i+1}+\frac{\alpha_{i}}{\varepsilon}\left(e_{1}-\widehat{e}_{1}\right), \quad 1 \leq i \leq r-1 \\
\hat{\hat{e}}_{n}=\frac{\alpha_{n}}{\varepsilon}\left(e_{1}-\hat{e}_{1}\right) \\
u=\frac{1}{g\left(\hat{e}+Y_{d}\right)}\left(y_{d}^{(r)}-K \hat{e}-f\left(\hat{e}+Y_{d}\right)-\frac{1}{\left(1-\Delta_{1}\left(\hat{e}+Y_{d}\right)\right)} \frac{B^{T} P \widehat{e} \mu^{2}\left(\widehat{e}+Y_{d}\right)}{\left\|B^{T} P \hat{e}\right\| \mu\left(\hat{e}+Y_{d}\right)+\varepsilon_{1} \exp \left(-\varepsilon_{2} t\right)}\right)
\end{gathered}
$$

Theorem 6. Suppose that $\Delta_{1}(x)=\sup _{x \in R^{r}}\left|\delta_{1}(x)\right|<1$, consider system (22) under the robust state feedback controller (32), the closed-loop system have the following properties:

(1) The state of the closed-loop system is bounded.

(2) The output of the closed-loop system satisfy $\lim _{t \rightarrow \infty}\left|y-y_{d}\right|=0$.

We can prove Theorem 2 by following the proof line of Theorem 1, the detailed proof is omitted here.

\section{Simulation study}

The performance of the nonlinear robust output feedback controller (28) was tested through simulations. The values of the process parameters are shown in Table 3.

Table 3 Model parameters for the simulation study 


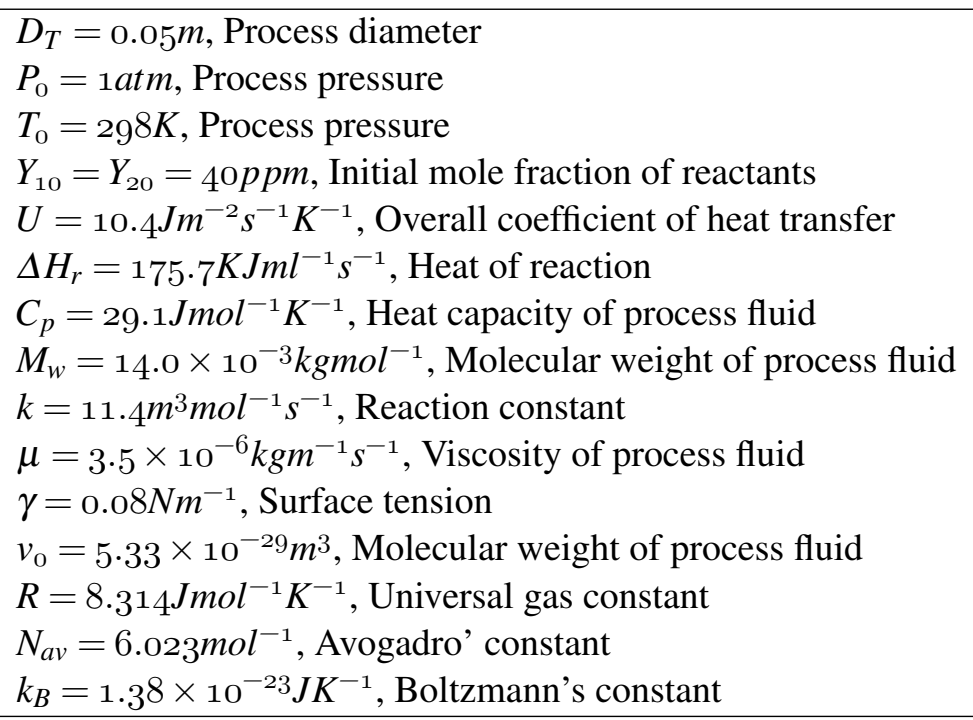

Several simulations were performed to evaluate the disturbance attenuation and set-point tracking capabilities of the robust nonlinear controller, as well as its robustness with respect to uncertainties in model parameters and unmodeled dynamics.

The objective of these simulations is to show that the use of proposed robust control allows producing an aerosol product with a desired geometric average particle diameter $\left(d_{p g}=0.18 \mu \mathrm{m}\right)$ within a given type of batch reactor even in the presence of uncertainty in the process model parameters. Figure 1 presents the closed-loop trajectory (dashed line) and closed-loop trajectory (solid line) for $d_{p g}$ under robust nonlinear controller and nonlinear controller, respectively, and the corresponding manipulated input trajectory in the case of $10 \%$ error in the value of the rate constant $\mathrm{k}$. It is clear that the robust nonlinear control regulates the output $d_{p g}$ to the desired point value (i.e. $d_{p g}=0.18 \mu \mathrm{m}$ ), and attenuate the effect of timevarying uncertainty on the process. However, nonlinear control cannot guarantee the output $d_{p g}$ to the desired point values in the presence of uncertainty. There is an error between steady-state value and the desired point one. Figure 2 presents the closed-loop trajectory (dashed line) and closed-loop profile (solid line) for $d_{p g}$ under robust nonlinear controller and nonlinear controller, and the corresponding manipulated input trajectory in the case of a $8 \%$ decrease in the parameters. Again, the robust nonlinear controller allows achieving an aerosol product with the desired $d_{p g}$.

Also we simulated the robustness properties of the proposed controller with respect to parametric model uncertainty and unmodeled dynamics in the presence of a set-point change, the robust nonlinear controller was found to have very good robustness properties, keeping the output on the set-point.

Remark 7. It is worth to point out that the proposed approach for the design of robust nonlinear controllers is applicable to most aerosol systems for which the hypothesis of unimodal lognormal aerosol size distribution for long times is valid.

\section{Conclusions}

In this paper, we present a comprehensive study on robust control of particle size distribution of fractal agglomerate in aerosol processes with simultaneous chemical reaction, nucleation, condensation and coagulation. Initially, a general aerosol process is presented by population balance and mass and energy balances, which describes the evolution of particle size distribution, continuous phase species and temperature of the aerosol system, respectively. A lognormal moment approximations of the population balance model is then presented. Then, the robust state feedback controller is designed for the aerosol system with some unknown uncertainties, the proposed controller is composed of a robust control term and an nominal control term so that it only ensures the stability of the closed-loop system, but also atten- 


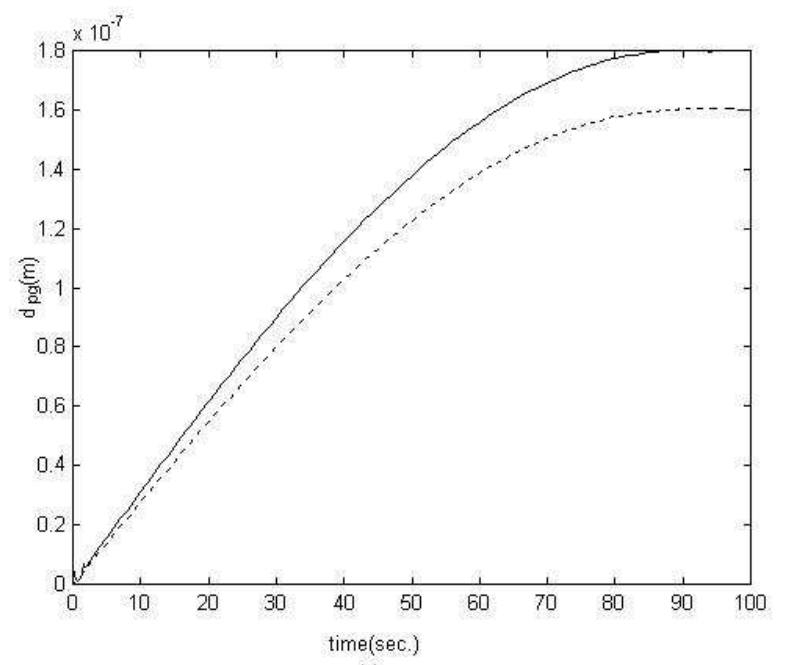

(a)

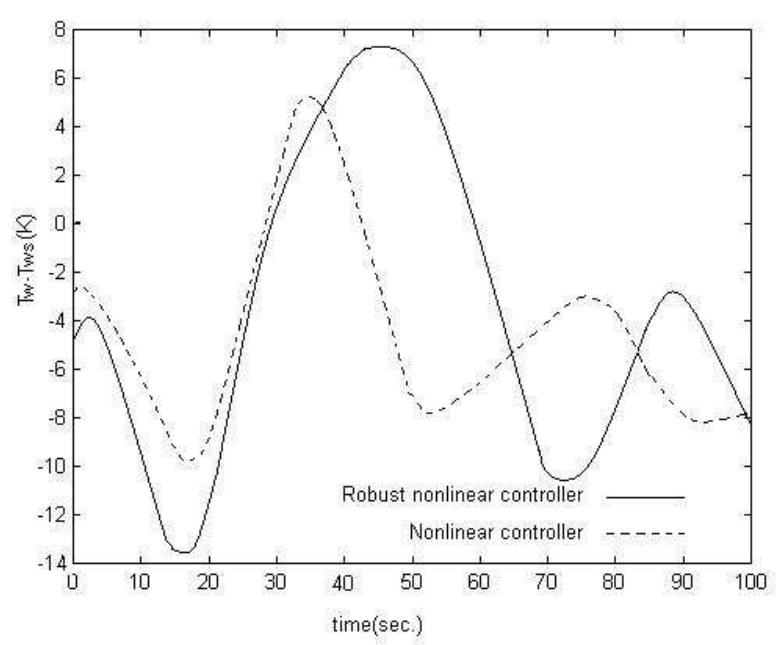

(b)

Figure 1: (a) $d_{p g}$ using the robust nonlinear controller and nonlinear controller, (b) The corresponding manipulated input trajectory

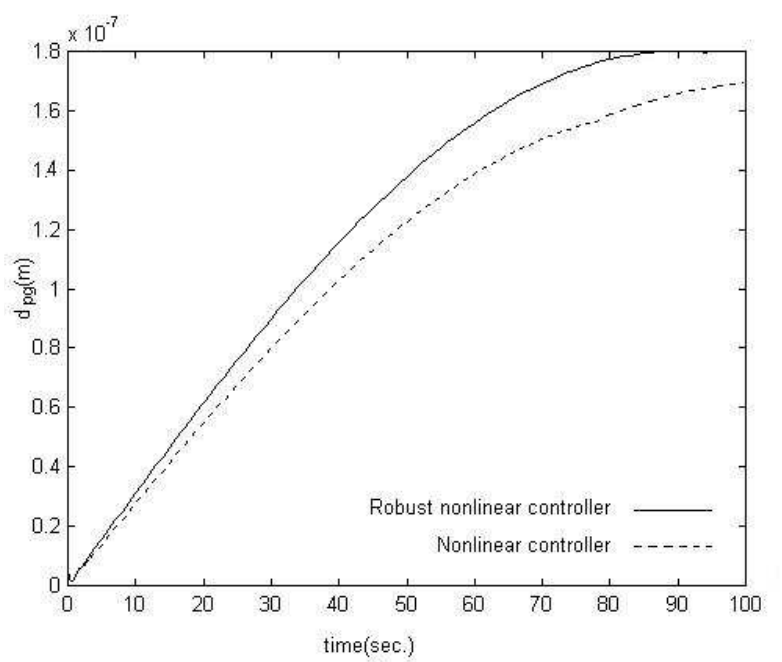

(a)

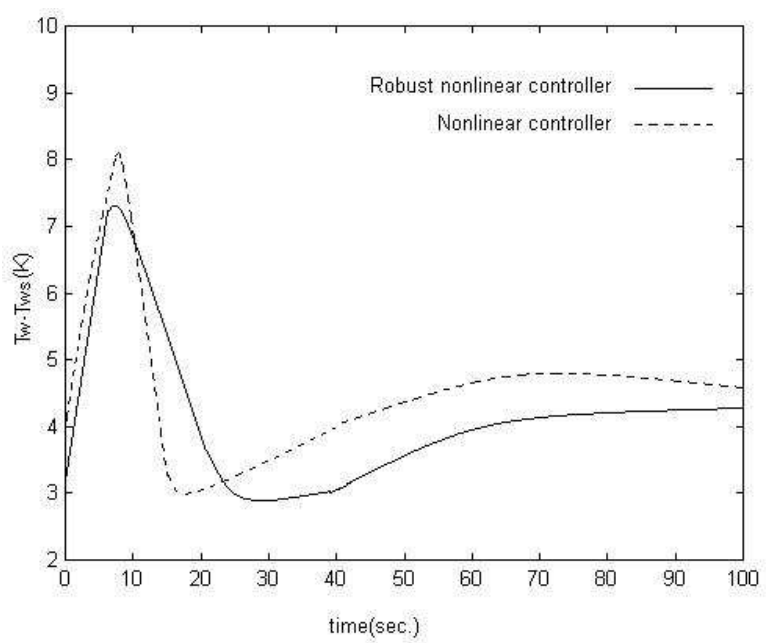

(b)

Figure 2: (a) $d_{p g}$ using the robust nonlinear controller and nonlinear controller, (b) The corresponding manipulated input trajectory 
uates the effect of the unknown uncertainties on the system. A high-gain observer is adopted to estimate state variables required in the on-line implementation of the state feedback. Finally, the proposed robust controller is applied to an aerosol process for achieving an aerosol size distribution with desired geometric average particle diameter, the simulation results show the robustness properties of the controller with respect to parametric model uncertainty and unmodeled dynamics.

\section{Acknowledgment}

This work is supported by the National Natural Science Foundation of China (No. 60974027).

\section{Bibliography}

[1] J. M. Hidy and J. R. Brock, The Dynamic of Aerocolloidal System, Oxford, Pergamon Press, 1970.

[2] V. M. Voloshuk, and Y. S. Sedunov, Coagulation phenomena in disperse systems, Moscow: Moskovskiy Inghenerno Fizicheskiy Institut, 1975 (in Russia)

[3] V. N. Piskunov, Physical phenomena in disperse systems, Leninggrad: Gidrometeoizdat Publishers, 1991 (in Russia)

[4] M. J. Hounslow, A discretized population balance for continuous systems at steady-state. American Institute of Chemical Engineers Journal, 36, pp. 106-116,1990

[5] P. J. Hill, and K. M. Ng, New discretization procedure for the breakage equation. American Institute of Chemical Engineers Journal, 41 , pp. 1204-1216, 1995

[6] P. J. Hill, and K. M. Ng, New discretization procedure for the agglomeration equation. American Institute of Chemical Engineers Journal, 42, pp. 727-741, 1996

[7] N. V. Mantzaris, P. Daoutidis, and F. Srienc, Numerical solution of multivariable cell population balance models. Parts: I, II and III. Computers and Chemical Engineering, 25, pp. 1411-1481, 2001

[8] M. Nicmanis, and M. J. Hounslow, Finite-element methods for steady-state population balance equations. American Institute of Chemical Engineers Journal, 44, pp. 2258-2272, 1998

[9] J. D. Landgrebe, and S. E. Pratsinis, A discrete sectional model for particulate production by gas phase chemical reaction and aerosol coagulation in the free molecular regime. Journal of Colloid Interface Science, 139, pp. 63-86, 1990

[10] E. Otto, H. Fissan, S. H. Park, and K. W. Lee, The Log-Normal Size Distribution Theory of Brownian Aerosol Coagulation for the Entire Particle Size Range. Part I: Analytical Solution Using the Harmonic Mean Coagulation Kernel, Journal of Aerosol Science, Vol. 30. No. 1 pp. 3-16, 1999

[11] S. H. Park, and K. W. Lee, Analytical Solution to change in size distribution of polydisperse particles in closed chamber due to diffusion and sedimentation, Atmospheric Environment, Vol. 36. pp. 5459-5467, 2002

[12] S. H. Park, and K. W. Lee, Change in particle size distribution of fractal agglomerates during Browian coagulation in the free-molecule regime, Journal of Colloid and Intreface Science, Vol. 246. pp. 85-91, 2002 
[13] V. N. Piskunov, and A. I. Golubev, The Generalized Approximation Method for Modelling Coagulation Kinetics "C Part I: Justification and Implementation of the Method, Journal of Aerosol Science , Vol. 33, pp. 51-63,2002

[14] P. Daoutidis, and P. D. Christofides, Dynamic feedforward/output feedback control of nonlinear processes, Chemical Engineering Science, Vol. 50, pp. 1889-2007, 1995

[15] A. Kalani, and P. D. Christofides, Nonlinear control of spatially-in homogeneous aerosol processes, Chemical Engineering Science, Vol. 54, pp. 2669-2678, 1999

[16] T. J. Crowley, E. S. Meadows, E. Kostoulas, and F. J. Doyle III, Control of Particle Size Distribution Described by a Population Balance Model of Semibatch Emulsion Polymerization, Journal of Process Control, Vol.10, 419-432, 2000.

[17] D. Semino, and W. H. Ray, Control of systems described by population balance equations-II. Emulsion polymerization with constrained control action, Chemical Engineering Science, Vol.50, pp. 1825-1839, 1995.

[18] T. Chiu, and P. D. Christofides, Nonlinear control of particulate processes, American Institute of chemical Engineers Journal,Vol. 45, pp. 1279-1297, 1999.

[19] N. H. El-Farra, T. Chiu, and P. D. Christofides, Analysis and control of particulate processes with input constraints, American Institute of Chemical Engineering Journal, 47, pp. 1849-1865, 2001.

[20] A. Kalani, and P. D. Christofides, Modeling and control of a titania aerosol reactor, Aerosol Science and Technology, 32, pp. 369-391, 2000.

[21] A. Kalani, and P. D. Christofides, Simulation, estimation and control of size distribution in aerosol processes with simultaneoue reaction, nucleation, condensation and coagulation, Computers and Chemical Engineering, 26, pp. 1153-1169, 2002.

[22] S. K. Friedlander, Smoke, Dust and Haze: Fundamentals of Aerosol Dynamics, New York: Oxford University Press, 2000.

[23] S. E. Pratsinis, Simultaneous nucleation, condensation, and coagulation in aerosol reactors, Journal of Colloid Interface Science, 124, pp. 416-426, 1988.

[24] A. Isidori, Nonlinear control systems: an introduction (Second Edition). Berlin, Heidelberg, Springer-verlag, 1989.

[25] E. Yaz, Stabilizing compensator design for uncertain nonlinear systems, Systems and Control Letter, 21, pp.11-17, 1993

Zhengrong Xiang was born in China, in 1969. He obtained his Ph.D. degree in Control Theory and Control Engineering from the Nanjing University of Science and Technology, China, in 1998. Since 2001, he has been an associate professor in the School of Automation. He is an IEEE member. His main research interests include nonlinear control, robust control, intelligent control, and switched systems. He has published significantly on the subjects with over 80 technical papers in journals and conferences. E-mail: xiangzr@mail.njust.edu.cn. 